\title{
Detection and quantification of intergenic transcription in Mycoplasma hyopneumoniae
}

Correspondence

F. Chris Minion

fcminion@iastate.edu

Received 3 February 2010

Revised 12 April 2010

Accepted 22 April 2010

\author{
Stuart W. Gardner ${ }^{1,2}$ and F. Chris Minion ${ }^{1}$
}

\author{
${ }^{1}$ Department of Veterinary Microbiology and Preventive Medicine, Interdepartmental Microbiology \\ Program, lowa State University, Ames, IA 50011, USA \\ ${ }^{2}$ Department of Statistics, lowa State University, Ames, IA 50011, USA
}

\section{INTRODUCTION}

Mycoplasmas are members of the class Mollicutes, cell-wallless bacteria that infect a wide variety of plants and animals (including humans). Though related taxonomically, members of the Mollicutes differ significantly in their habitats, their growth requirements and their overall structure. They are believed to have evolved from more complex organisms through degenerative mechanisms with the concomitant loss of genetic material (Neimark, 1986); all mycoplasmas have small genomes with a limited number of genes, resulting in a lack of biosynthetic pathways (Hutchison \& Montague, 2002; Pollack et al., 1997). Thus, they must obtain amino acids, purines, pyrimidines and membrane components from their growth environment. For the members of the family Mycoplasmataceae, the animal pathogens, this occurs primarily at the host mucosal

Abbreviations: IG, intergenic; qRT-PCR, quantitative real-time-PCR.

The microarray descriptions and the microarray data can be accessed through the Gene Expression Omnibus (http://www.ncbi.nlm.nih.gov/ geo) under accession number GSE17893.

A set of supplementary methods, four supplementary figures and five supplementary tables are available with the online version of this paper. surface, where damage to host cells occurs through different mechanisms (Razin \& Herrmann, 2002).

Mycoplasmas are found in association with hosts and not in a variety of external environments like many other pathogenic bacteria. Thus, the question arises as to whether mycoplasmas need to regulate genes if their environments are restricted to host mucosal surfaces, and if they do, then by what mechanisms. It has been suggested that with the loss of biosynthetic pathways, the remaining genes that are responsible for the basic processes of the cell, i.e. DNA replication, transcription and translation, are thought to be constitutively expressed, and in constant environments, the organism supposedly has little need for sophisticated genetic control mechanisms (Muto \& Ushida, 2002). Our knowledge of gene expression in mycoplasmas is rudimentary, with few studies that actually address the mechanisms involved (Benders et al., 2005; Chang et al., 2008; Güell et al., 2009; Lluch-Senar et al., 2007; Weiner et al., 2000, 2003). Transcription is essential to the basic process of gene expression, but surprisingly, little information has been obtained experimentally defining the transcription process in mycoplasmas (Muto \& Ushida, 2002). The heat-shock response has been studied in a 
limited number of mycoplasma species (Madsen et al., 2006b; Musatovova et al., 2006; Weiner et al., 2003), and it is clear that a transcriptional activator, HrcA, is involved in regulating transcription of two heat-shock genes (Chang et al., 2008). In addition, transcriptional start sites have been mapped for a limited number of genes (Hyman et al., 1988; Taschke \& Herrmann, 1988; Taschke et al., 1986; Weiner et al., 2000), and promoter-probe vectors have been used in several different species (Janis et al., 2005; Knudtson \& Minion, 1993, 1994; Lluch-Senar et al., 2007). These studies show that the -10 box of the Pribnow sequence is relatively conserved, but not the -35 sequence. This lack of progress has been partly due to the few genetic tools available for use with mycoplasmas and with some species, e.g. Mycoplasma hyopneumoniae, the total lack of such tools. The limited genome capacity suggests simple regulatory mechanisms, and experimental evidence indicates that mycoplasmas have few regulatory systems for several reasons: (1) only a single sigma factor has been identified in any of the complete mycoplasma genome sequences, (2) transcriptional activators have rarely been identified (Chang et al., 2008), and (3) the genes for a few repressor-like proteins have been identified in genome sequence studies (Fraser et al., 1995; Glass et al., 2000; Minion et al., 2004). The global assessment of transcription has been reported using arrays (Madsen et al., 2006a, b, 2008; Oneal et al., 2008; Schafer et al., 2007; Weiner et al., 2003), but these tools are limited because they only assess transcriptional changes in annotated gene sequences and do not address mechanisms of transcription control.

The subject of this study, M. hyopneumoniae, is associated with respiratory disease and reduced productivity in swine (Ross, 1992). It is the causative agent of porcine enzootic pneumonia, and when co-infections occur with a secondary (bacterial or viral) infection, the respiratory disease is more severe and has been designated porcine respiratory disease complex (Ross, 1992). Even though the virulence of $M$. hyopneumoniae appears to be low, substantial economic losses occur as a consequence of reduced average daily weight gain and efficiency of feed utilization, prophylactic and therapeutic costs, and mortality (Pointon et al., 1985; Ross, 1992). The virulence factors of M. hyopneumoniae are largely unknown, and to better understand the mechanisms involved, we are studying transcription in M. hyopneumoniae both in vitro and in vivo (Adams et al., 2005; Madsen et al., 2006a, b, 2008; Oneal et al., 2008; Schafer et al., 2007). Recent studies using microarrays to examine global transcriptional changes clearly show that $M$. hyopneumoniae can regulate its genes in response to environmental changes (Madsen et al., 2006a, b, 2008; Oneal et al., 2008; Schafer et al., 2007). These studies give a snapshot of transcription across the genome in the annotated genes, but they cannot reveal basic features of transcription, i.e. start and stop sequences and mechanisms of regulation.

Many mycoplasma genes lack defined, typical or canonical transcriptional stop sequences; rho-dependent termination does not seem to operate in mycoplasmas (Razin et al., 1998) and rho-independent sequences cannot often be found. To better understand transcription termination in mycoplasmas and as a first step in defining potential regulatory RNAs that may serve a role in gene regulation (Gottesman, 2005; Szymanski et al., 2003; Waters \& Storz, 2009), we sought to determine if intergenic (IG) regions were transcribed in $M$. hyopneumoniae. The genome of $M$. hyopneumoniae consists of 698 protein-coding ORFs and 35 rRNA genes, and due to overlapping and juxtaposed ORFs there are 573 IG regions (Minion et al., 2004). To complicate the study, the overall $\mathrm{G}+\mathrm{C}$ content of the genome is $28.6 \mathrm{~mol} \%$, with ORFs having $29.6 \mathrm{~mol} \%$ and IG regions $18.7 \mathrm{~mol} \% \mathrm{G}+\mathrm{C}$. Surprisingly, the results of this study indicate that transcripts specific to virtually all of the 343 IG regions longer than 50 bases can be identified in M. hyopneumoniae RNA preparations. In a more defined quantitative analysis of five IG regions, it was clearly shown that internal initiation of transcription in IG regions occurs and probably does so at high frequency, suggesting that these RNAs may play an important role in the physiology of the mycoplasma, such as acting as regulatory RNAs. To our knowledge this is the first study to assess transcription across IG regions of the $M$. hyopneumoniae genome. A recent report by Güell et al. (2009) also demonstrated IG transcription in Mycoplasma pneumoniae, suggesting that transcripts arising from IG regions are common in mycoplasmas.

\section{METHODS}

Mycoplasma strains and culture conditions. Pathogenic $M$. hyopneumoniae strain 232, a derivative of strain 11, was used in this study (Mare \& Switzer, 1965). Cultures were passed fewer than 15 times in vitro in Friis medium (Friis, 1975). For the microarray and IG transcriptional studies, three $250 \mathrm{ml}$ flasks containing $125 \mathrm{ml}$ cultures were grown at $37{ }^{\circ} \mathrm{C}$ to early exponential phase, as determined by medium colour change and optical density. Cells were pelleted by centrifugation at $24000 \mathrm{~g}, 500 \mu \mathrm{l}$ RNAlater (Ambion) was added, and pellets were stored at $-70{ }^{\circ} \mathrm{C}$ until the total RNA was isolated.

For the heat-shock study, six cultures of $5.6 \mathrm{ml}$ were grown to exponential phase as described previously (Madsen et al., 2006b). Three cultures were randomly chosen and then shifted to $42{ }^{\circ} \mathrm{C}$ for $2 \mathrm{~h}$ while the other three cultures were maintained at $37{ }^{\circ} \mathrm{C}$ for these $2 \mathrm{~h}$. Cells were then pelleted and stored as described above until total RNA isolation.

RNA isolation. RNA was isolated from frozen cell pellets using Ambion's RiboPure kit according to the manufacturer's protocol. Each sample was treated with $4 \mathrm{U}$ DNase at $37{ }^{\circ} \mathrm{C}$ for $30 \mathrm{~min}$ followed by purification using a Millipore Ultracell YM-30 Microcon Centrifugal Filter unit. Quantification and purity checks of each sample were done using a Nanodrop ND-1000 spectrophotometer. RNA samples were confirmed to be DNA free via PCR and gel electrophoresis.

Microarrays. Two types of microarrays were constructed, one consisting of PCR products and the other of synthesized oligonucleotides. Intergenic regions were defined as non-coding DNA sequences between annotated genes in the M. hyopneumoniae genome sequence (Minion et al., 2004). Only IG regions larger than $49 \mathrm{bp}$ were considered for analysis, and those greater than $124 \mathrm{bp}$ were considered for PCR-based probes. Oligonucleotides were used for the smaller IG regions and for sequences that failed in PCRs to complete the coverage 
(defined as $>50 \%$ of the IG region). PCR primers were designed for products of $125-450 \mathrm{bp}$ using the Primer3 online software program (Rozen \& Skaletsky, 2000). PCRs were performed in 96-well plate format in a MJ Research Dyad thermocycler in $100 \mu$ reactions using the following conditions: $1 \times \mathrm{MgCl}_{2}$-free PCR buffer (Invitrogen); $3.5 \mathrm{mM} \mathrm{MgCl} 2 ; 0.2 \mathrm{mM}$ each of dATP, dTTP, dGTP and dCTP; 200 pmol of each primer; 2 units Taq polymerase (Invitrogen); and $100 \mathrm{ng}$ M. hyopneumoniae strain 232 chromosomal DNA obtained via phenol/chloroform extraction and ethanol precipitation. Thermocycler conditions started with denaturation at $95{ }^{\circ} \mathrm{C}$ for $5 \mathrm{~min}$ followed by 30 cycles of denaturation at $94{ }^{\circ} \mathrm{C}$ for $1 \mathrm{~min}$, annealing at $56{ }^{\circ} \mathrm{C}$ for $1 \mathrm{~min}$, and elongation at $68{ }^{\circ} \mathrm{C}$ for $30 \mathrm{~s}$, and ending with a final elongation at $68{ }^{\circ} \mathrm{C}$ for $10 \mathrm{~min}$. Products were confirmed by $1.2 \%$ agarose gel electrophoresis and purified via Mo Bio's UltraClean PCR Clean-Up kit according to the manufacturer's protocol. Purified PCR products were quantified by visual inspection, dried via vacuum centrifugation, and resuspended to approximately $200 \mathrm{ng} \mu \mathrm{l}^{-1}$ in Corning spotting buffer. Products suspended in spotting buffer were electrophoresed in $1.2 \%$ agarose gels to confirm purity and concentration of each product and its size. The PCR product microarrays were spotted to Corning UltraGAPS glass substrates in triplicate by the University of Iowa DNA facility.

Oligonucleotides were designed for some IG regions based on the inability to produce PCR products or the length of the region (49$124 \mathrm{bp}$ ). The strand chosen to be synthesized for the IG region probes was in the same orientation as the closest adjacent ORF. Oligonucleotides were synthesized by IDT and ranged in size between

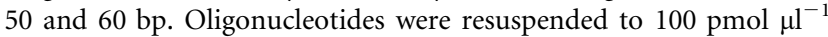
in nuclease-free water, and then $9 \mu \mathrm{l}$ were vacuum dried and diluted to $30 \mu \mathrm{M}$ in IDT Epoxide spotting buffer. These oligonucleotides were spotted to Corning Epoxide substrates in triplicate.

Microarray experimental design. To test for transcripts in IG regions, cDNA was generated from $M$. hyopneumoniae RNA using either random hexamers or a set of 129 hexamer oligonucleotide primers designed for M. hyopneumoniae mRNA (Madsen et al., 2006b). A single total RNA sample was split into eight $5 \mu \mathrm{g}$ samples, and cDNA synthesis was conducted on four of these samples using random hexamers (IDT); for the other four samples, cDNA was generated using primers designed for M. hyopneumoniae as described by Madsen et al. (2006b). In each set of four, two samples were fluorescently labelled with Cy3 and the other two were labelled with Cy5 using an indirect labelling protocol (Madsen et al., 2006b). After labelling and cleanup, all eight samples were quantified on a Nanodrop ND-1000 spectrophotometer. For each array on the slide, $1 \mu \mathrm{g}$ labelled cDNA from each type of primer generation ( $\mathrm{Cy} 3$ or Cy5, random or specific primer set) was combined and hybridized to a PCR-featured array and an oligonucleotide-featured array. This resulted in two arrays of PCR probes hybridized with dye swaps and two arrays of oligonucleotide probes with dye swaps. A second RNA sample was split into four $5 \mu \mathrm{g}$ samples of total RNA, and the hybridization process was repeated for the oligonucleotide probe array. The hybridization and wash protocols have been described for PCR (Madsen et al., 2006b) and oligonucleotide arrays [as per manufacturer's instructions (Corning)].

Microarray data acquisition and analysis. Slides were scanned using a ScanArray Express laser scanner as described previously (Madsen et al., 2006b). The SoftWorRx Tracker analysis software package (Applied Precision) was used to quantify the images. Probes were declared to detect signal if their normalized intensity in both channels on at least one array was more than 6 SEM greater than the mean intensity of features containing spot buffer only.

Microarray data accession number. The microarray descriptions and the microarray data can be accessed through the Gene Expression
Omnibus (http://www.ncbi.nlm.nih.gov/geo) under accession number GSE17893.

qRT-PCR primer design. The IG sequence and 300 bases into adjacent ORFs were targeted for relative quantitative real-time-PCR (qRT-PCR) analysis. Primers were designed using the IDT Primer Quest tool. The goal was to design six primer pairs for each region with unique target sequences ranging in size from 80 to $200 \mathrm{bp}$. One target sequence each was designed to reside completely within each flanking ORF, one each to bridge the transition region from ORF to IG region, and two within the IG region itself. For clarity, the IG regions were designated according to the upstream ORF (numbered clockwise around the genome), independent of transcription direction. Additional primers for the heat-shock assay were similarly designed for the region upstream of $d n a K$. This region of the genome does not contain a large IG region; thus primers were designed such that a target sequence was located in each gene ( $u v r C$, mhp071 and $d n a K)$; and three target sequences bridged genic/intergenic regions (uvrC/ig-070, mhp071/ig-071 and ig-071/dnaK). Additionally, primers for a target sequence within the reference gene control (mhp381) used in the analysis were designed. Table 1 describes the primers used for the qRTPCR analysis. All of the primers were tested using a M. hyopneumoniae chromosomal DNA template to ensure their effectiveness in producing a correct product as determined by size via agarose gel analysis.

qRT-PCR experimental design and data acquisition. A test of the sensitivity of the qRT-PCR assay was done using DNA from $M$. hyopneumoniae 232 diluted to $4 \mathrm{ng}^{-1} \mathrm{l}^{-1}$. The Express One-Step SYBR GreenER kit (Invitrogen) was used for qRT-PCR with the Mx3005P QPCR System (Stratagene) and MxPro 4.1 software. Reaction volumes for each well totalled $15 \mu \mathrm{l}$ and contained $0.895 \mu \mathrm{l}$ water, $7.5 \mu \mathrm{l} \mathrm{qPCR} \mathrm{mix,} 0.6 \mu \mathrm{l}$ of each primer at $2.5 \mu \mathrm{M}, 0.03 \mu \mathrm{l}$ ROX reference dye, $5 \mu \mathrm{l}$ template DNA or RNA at $4 \mathrm{ng}^{-1} \mathrm{l}^{-1}$ and $0.375 \mu \mathrm{l}$ SuperScript III. The DNA template was diluted 11 times as described by the PREXCEL-Q software program (Gallup \& Ackermann, 2006). These 11 dilutions and a negative control were run in a 96-well plate for the six target sequences in region ig-072. The thermal cycling conditions were a (mock) cDNA synthesis step at $50{ }^{\circ} \mathrm{C}$ for $30 \mathrm{~min}$; RT deactivation at $95{ }^{\circ} \mathrm{C}$ for $10 \mathrm{~min}$; 40 PCR cycles of $95{ }^{\circ} \mathrm{C}$ for $30 \mathrm{~s}$, $56{ }^{\circ} \mathrm{C}$ for $30 \mathrm{~s}$, and $68{ }^{\circ} \mathrm{C}$ for $30 \mathrm{~s}$; and a melt-curve step of $95{ }^{\circ} \mathrm{C}$ for $10 \mathrm{~min}, 55^{\circ} \mathrm{C}$ for $30 \mathrm{~s}$, and $95{ }^{\circ} \mathrm{C}$ for $30 \mathrm{~s}$. These thermal cycling conditions were used for all qRT-PCR assays throughout this study.

Test plates (Gallup \& Ackermann, 2006) were run on five qRT-PCR regions (ig-072, ig-106, ig-282, ig-682, ig-684) for detection of inhibition and primer specificity of qRT-PCR. Test plates used in the transcriptional analysis were conducted on a mixture of equal volumes of two M. hyopneumoniae strain 232 RNA samples (stock I)

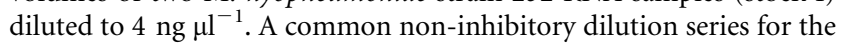
five or six target sequences within an IG region was determined separately for each region.

The qRT-PCR assays were conducted in a single 96-well plate on each of the two RNA samples in the non-inhibitory dilution range as determined in the test plates. The region ig-072 assay consisted of six primer sets and four dilutions per primer set. Each well condition was run in duplicate. Assays for regions ig-106 and ig-282 consisted of five primer sets and eight dilutions per primer set. Duplicate wells were run for primer set 3 in region ig-106 and for primer set 4 in region ig-282. Assays for regions ig-682 and ig-684 consisted of six primer sets and eight dilutions per primer set. No duplicate wells were run for these two regions.

Quantification cycle $(C q)$ values were obtained from the MxPro 4.1 software's default amplification-based threshold method. This method specifies the threshold as the mid-point of the exponential amplification phase across all amplification curves on the plate. For duplicate samples on an assay plate (technical replicates), the $C q$ values were averaged for subsequent analysis. 
Table 1. Primers used for qRT-PCR analysis

\begin{tabular}{|c|c|c|c|c|c|c|}
\hline Region & $\begin{array}{c}\text { Primer } \\
\text { set }\end{array}$ & Forward primer & Sequence & Reverse primer & Sequence & $\begin{array}{l}\text { Product } \\
\text { size (bp) }\end{array}$ \\
\hline ig-070/071 & 1 & RT_uvrC.L & TGCTCCTTTTGCATACTGATTC & RT_uvrC.R & AAAATTGATGCTGCCAAACC & 121 \\
\hline ig-070/071 & 2 & RT_uvrC-ig070.L & TTTGGCAGCATCAATTTTT & RT_uvrC-ig070.R & TCACTCTAATTTATTTTAACTTCAAGG & 150 \\
\hline ig-070/071 & 3 & RT_mhp071.L & TCTGTTCCTAAAATAAAACAGTTGAAA & RT_mhp071.R & CGATAAAATCATTTGGCAAGC & 142 \\
\hline ig-070/071 & 4 & RT_mhp071-ig071.L & AAAAGTTGAAAGAGATATTGAAACTCA & RT_mhp071-ig071.R & TGCCAGAATTTATGAAAAATAGTCA & 126 \\
\hline ig-070/071 & 5 & RT_ig071-dnaK.L & TTTTTCATAAATTCTGGCACTTTT & RT_ig071-dnaK.R & CCTAAAATGATTTCTTTTGCCATT & 147 \\
\hline ig-070/071 & 6 & RT_dnaK.L & CCTGTCGTTCTCGAAAATCC & RT_dnaK.R & CCCCGACAATTTCTTCATTG & 82 \\
\hline ig-072* & 1 & RT_mhp072.F & ACAGTTCGTGCCGAAGGGCTTATT & RT_mhp072.R & TTGGGCAGCTGCTTCAATTTGGTC & 183 \\
\hline ig-072* & 2 & RT_mhp072.ig1.F & AACCGCGCAGCAAGCAAATACATC & RT_mhp072.ig1.R & CTAGCAGAAATTTGCAAGTGTGCGT & 129 \\
\hline ig-072* & 3 & RT_mhp072.ig2.F & AAGAAATCCAGGAAGCCTACGA & RT_mhp072.ig2.R & AAGCCACTAGACCCACCACCAAAT & 112 \\
\hline ig-072* & 4 & RT_mhp072.ig3.F & TTTGGTGGCTCACAAGAACAAA & RT_mhp072.ig3.R & CGCCTGAACCCTTACAGTTATCACA & 157 \\
\hline ig-072* & 5 & RT_mhp072.ig4.F & GATAACTGTAAGGGTTCAGGCGCT & RT_mhp072.ig4.R & TGAACCCGACCAAATCCGGGAATA & 116 \\
\hline ig-072* & 6 & RT_mhp073.F & TATTCCCGGATTTGGTCGGGTTCA & RT_mhp073.R & AAACATTCCATCCTGGATTCCGGC & 166 \\
\hline ig-106 & 1 & RT_mhp106.F & TGAGTTGCAAGCAATTGATTATCGC & RT_mhp106.R & CCATATTCCСТTAATTTAATTTCAAGTTTGC & 200 \\
\hline ig-106 & 2 & RT_mhp106.ig2.F & TGTCTTCAAGTTAGACTTGCTTAT & RT_mhp106.ig2.R2 & GGATAATTTAGGTGATTTCATGTTATT & 123 \\
\hline ig-106 & 3 & RT_mhp106.ig3.F & ACATGAAATCACCTAAATTATCCT & RT_mhp106.ig3.R & AGAAGCCACTAAATTATTCAAGA & 82 \\
\hline ig-106 & 4 & RT_mhp106.ig4.F & AATTGTAAATTCGCGGAGGTGAAC & RT_mhp106.ig4.R & CCCGCGGGTGAGAACCATTATATTT & 170 \\
\hline ig-106 & 5 & RT_mhp107.F & GCAGGCTAATTTGATTGGCAGA & RT_mhp107.R & CCGCGGGTGAGAACCATTATATTT & 139 \\
\hline ig- $282^{*}$ & 1 & RT_mhp282.F & CCATAGTAAGAGCATAAATTGGAACCC & RT_mhp282.R & CCACCGGATTTAATTTCAGAACGCCC & 91 \\
\hline ig- $282^{*}$ & 2 & RT_mhp282.ig2.F & GATGGATGTAAGTGAGTTTGGGCA & RT_mhp282.ig2.R & TTTCCCGACAAAGTGGCTCT & 88 \\
\hline ig- $282^{*}$ & 3 & RT_mhp282.ig3.F & ATTAGAGCCACTTTGTCGGG & RT_mhp282.ig3.R & AATTAGATTCTCCAATCGATCAAA & 115 \\
\hline ig-282 & 4 & RT_mhp282.ig4.F & GCACGTAATTTATAGTGCTTT & RT_mhp282.ig4.R & GCCGTTGAGTCTAATTCAGATG & 153 \\
\hline ig-282 & 5 & RT_mhp283.F & ACTGCGGAGACGATAATCTGA & RT_mhp283.R & TCCTTGGACCTTCCTTCTGCGATT & 90 \\
\hline ig-682 & 1 & RT_mhp682.F2 & AGTGACTCAAAGTTAGTGAAGCA & RT_mhp682.R & ATAAAGTCGCTGTCTGGG & 199 \\
\hline ig- $682^{*}$ & 2 & RT_mhp682.ig1.F & ACAATTACAATCAAAGGCGAAATGC & RT_mhp682.ig1.R & GTTATGGTGTCCGCACTCGAAACT & 142 \\
\hline ig- $682^{*}$ & 3 & RT_mhp682.ig2.F & TCTCTGCTTAAAGAGCAATTGGA & RT_mhp682.ig2.R & GGGCTGAATTGTTGTGGGTAGT & 179 \\
\hline ig-682 & 4 & RT_mhp682.ig3.F2 & CAGCCCCAACCAATCAAATA & RT_mhp682.ig3.R & CTAATAACTGTAAGAGTCAAG & 118 \\
\hline ig- $682^{*}$ & 5 & RT_mhp682.ig4.F & TCATTCTACCAGAAATTAGA & RT_mhp682.ig4.R & GGGCTATTTAGGGAATAAA & 170 \\
\hline ig-682* & 6 & RT_mhp683.F & TCGTCTTGGGCTTGGGTATTGGTA & RT_mhp683.R & ATGATAGCTCTGATGAGCCTGAGC & 136 \\
\hline ig- $684^{*}$ & 1 & RT_mhp684.F & AAACTTTCAAGTTCAGCGCGGG & RT_mhp684.R & ACAGCCATTGTCGGAGTTGCAGTA & 98 \\
\hline ig-684 & 2 & RT_mhp684.ig1.F & ACAATGGCTGTTGCTGTTAC & RT_mhp684.ig1.R & AACCAATCAAGAAATTTCAGAACA & 194 \\
\hline ig- $684^{*}$ & 3 & RT_mhp684.ig2.F & AACAATAGTTGACAACCAAA & RT_mhp684.ig2.R & AATGAGGGTTAAACCAA & 116 \\
\hline ig- $684^{*}$ & 4 & RT_mhp684.ig3.F & GGTTTAACCCTCATTATTTATTAC & RT_mhp684.ig3.R & TTGTGTTATAACTCGGAATTT & 135 \\
\hline ig-684 & 5 & RT_mhp684.ig4.F2 & CCTAGGAGGTTCATATGCGTTT & RT_mhp684.ig4.R & GGTTAGAAATCCCGC & 104 \\
\hline ig-684 & 6 & RT_mhp685.F & AAAGAGGCGGGATTTCTAACCCA & RT_mhp685.R & ATATCGCTTAGTTTATTGCGTACTTCC & 200 \\
\hline Reference & 1 & RT_mhp381.L2 & TGACTAAATTCGGGCAATCC & RT_mhp381.R & ACTGTCGAATCGGTGAGGC & 172 \\
\hline
\end{tabular}

${ }^{*}$ Indicates primers were confirmed with DNA template in qRT-PCR. 
qRT-PCR relative quantity index. A simple linear model of $C q=m x+b$, where $C q$ is the quantification cycle and $x=\log _{2}(z)$, where $z$ is the dilution factor, was fitted to the data of each primer set in an assay. Note that the dilution factor $z$ (and $x$ ) is inversely proportional to the concentration of target RNA in the sample; as the sample dilution increases, the concentration of the target sequence RNA decreases. A standard curve is constructed by estimating $m$ and $b$ from the data of a given dilution series. Extrapolating this standard curve to $C q=0$, the cycle preceding PCR where a sample would be at the detection level, the model becomes $0=m x+b$. Solving for $x$ results in $x=-b / m$. This quantity is a relative measure of the initial amount of target sequence RNA in the sample, but since $x$ is inversely related to relative quantity, $-b / m$ is also inversely related to relative quantity. Thus, a lower $-b / m$ corresponds to more target RNA in the sample. An index that would be more intuitive is the negative of $-b / m$, namely $b / m$. This index is directly related to relative quantity. That is, the higher the $b / m$, the more target sequence RNA there is in the sample. A derivation of this relative quantity may also be seen as follows: $b / m=-x=-\log _{2}(z)=\log _{2}\left(z^{-1}\right)=\log _{2}(Q)$, where $Q$ is the relative quantity measure of the target RNA in a sample. An example calculation of the relative quantity index is available with the online version of this paper (Supplementary Methods: example calculation of the relative quantity index).

qRT-PCR DNA assay analysis. A single plate and sample DNA was used in the assay. Separate linear models were fitted to each primer set to obtain estimates of $m$ and $b$. These estimates were used to estimate the relative quantity index $(\mathrm{b} / \mathrm{m})$, and the delta method (Casella \& Berger, 2002) was employed to obtain an estimate of the SE of these quantity indices. All pairwise comparisons of the six primer set indices were done with a Bonferroni correction for multiple testing.

qRT-PCR transcript analysis. For the IG region assays with two RNA samples, the simple linear model above was extended to a linear model with a separate mean for each RNA sample: $C q=m x+b_{i}$, where $b_{i}$ is the intercept for RNA sample $i$. Thus, the model assumes that the two RNA samples have the same slope $(m)$ but different intercepts. For primer sets that had technical replications, the linear model was fitted to the averaged $C q$ values. Separate linear models were fitted to each primer set to obtain estimates of $m$ and $b_{i}$ for each RNA sample. The relative quantity index $(b / m)$ was estimated from these results and the delta method was employed to obtain an estimate of the SE of these quantity indices. All pairwise comparisons of the six primer set indices were done with a Bonferroni correction for multiple testing. To identify stem-loop structures in the ig-072, ig-106, ig-282, ig-682 and ig-684 IG regions, SoftBerry FindTerm software was used.

Heat-shock experimental design and data analysis. The upstream region (ig-070/071) and downstream region (ig-072) of dnaK served as a positive control since previous studies have shown that $d n a K$ is upregulated during heat shock (Madsen et al., 2006b). Six RNA samples, three control and three heat shock, were used in the assays. Test plates for the RNA samples and primer sets for both regions on either side of $d n a K$ were conducted on equal volumes of three heat-shock RNA samples and three control RNA samples

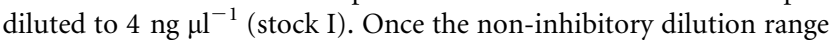
for each region had been determined across the six target sequences in each region, the qRT-PCR assay was run in duplicate. Half of an assay plate consisted of six dilutions of stock I (individual wells for the target sequences and duplicates for the reference gene) in order to construct the standard curves. The other half of the assay plate consisted of each RNA sample combined with the six primer sets run individually and a seventh primer set for the reference gene (mhp381) run in duplicate. In the downstream region (ig-072), the three heatshock RNA samples and three control RNA samples were diluted $1: 3$ for an in-well dilution of $1: 9$; and for the region upstream of $d n a K$ (ig-070/071), these samples were diluted 1:5 for an in-well dilution of $1: 15$. These sample dilutions corresponded near to the centre of the stock I dilution series as determined from the test plates for each region.

The standard curves for each probe set were calculated from the stock I dilution series by fitting the linear model $(C q=m x+b)$ to obtain estimates of $m$ and $b$ for each primer set per plate. The observed $C q$ values for the RNA samples were then normalized $\left(C q^{\prime}\right)$ by the reference gene (see Supplementary Methods for an example calculation). After normalization, the relative quantity of a primer set was then calculated as $C q^{\prime} / m$. This relative quantity is a linear shift of the relative quantity index $b / \mathrm{m}$. The derivation is easily seen by recalling that the linear model is $C q=m x+b$, so $C q / m=(m x+b) / m$ $=x+b / m$. Thus, for each target sequence there are two sets of three relative indices, one set for the control samples and the other set for the heat-shock samples. These indices are then averaged within treatment groups and the SEM for each target sequence/treatment combination is calculated. Fold change was calculated as described by the ISU equation of Gallup \& Ackermann (2006).

\section{RESULTS}

\section{Microarray design}

An analysis of the IG regions of the annotated $M$. hyopneumoniae strain 232 genome (Minion et al., 2004) identified 215 IG regions of $125 \mathrm{bp}$ or more and 128 IG regions of 50-124 bp for a total of 343 IG regions (see Supplementary Fig. S1). The remaining 230 IG regions of less than $50 \mathrm{bp}$ were not analysed since previous studies have shown that regions this small could serve to separate ORFs in an operon from which a polycistronic mRNA is produced (Adams et al., 2005). A total of 158 PCRgenerated probes for 112 of the 215 IG regions larger than $125 \mathrm{bp}$ were designed. Some of the larger regions had more than one probe designed to achieve at least $50 \%$ coverage. An additional 517 oligonucleotides for 247 IG regions were designed covering 128 IG regions of 50-124 bp and 119 IG regions of $125 \mathrm{bp}$ or more for which PCR primers could not be designed due to the high $\mathrm{A}+\mathrm{T}$ content of the IG regions $(81.3 \mathrm{~mol} \%)$ or a failure of PCRs (after repeated primer designs) for unknown reasons. A total of 16 IG regions had both PCR and oligonucleotide probes constructed.

\section{Microarray IG region probe detection}

Since mycoplasmal mRNAs lack polyadenylated sequences, we sought the most efficient primer set for producing cDNAs from RNA preparations. Our choices were a full random primer set of hexamers and a subset of 129 hexamer primers shown to bind to protein-encoding $M$. hyopneumoniae ORFs used in previous microarray studies, and listed in Supplementary Table S3 of Madsen et al. (2006b). We compared the two using the same RNA preparation at identical concentrations in reverse transcriptase reactions to generate cDNA for subsequent labelling reactions. Our hypothesis was that the random primers would be more efficient in producing cDNAs from 
IG RNAs than the subset of primers selected for ORF sequences and consequently produce stronger signals on the IG arrays. One caveat that might confound that hypothesis is the fact that we would be labelling rRNAs as well. A visual inspection of both arrays following hybridization with the two labelled cDNA preparations showed no appreciable difference in probe fluorescence between the two types of primers used to generate cDNA (data not shown). Therefore, the data from both primer sets were used in the analysis of the array features.

Applying the detection criterion (more than 6 SEM above the mean intensity of spot buffer-only probes in both channels) to the scanned arrays resulted in most of the IG regions declared as being transcribed. For the PCR probe array 94 out of $112(83.9 \%)$ IG regions had positive signals, and for the oligonucleotide array 239 out of 247 $(96.8 \%)$ IG regions had positive signals. Not all probes within an IG region had a positive signal, but for a region to be declared positive, at least one probe within a region was positive. Since 16 IG regions had both kinds of probes, of which 12 detected positive signals, this resulted in a total of 321 (94 PCR + 239 oligonucleotide -12 double counted) out of the 343 IG regions (93.6\%) with positive signals across both array types. Interestingly, of the 73 IG regions with outwardly divergent transcription, $68(93.2 \%)$ had positive signals. Those IG regions with inward transcription from both ORFs had $98 \%$ positive signals (52/53), and the IG regions with at least one inward transcript had $92.6 \%$ (201/217) positive signals (see Supplementary Table S1). Analysis of five IG regions for stem-loop structures identified potential stem-loops in ig-072 at the end of mhp072 and in ig-682 at the end of mhp683 (Fig. 1b, e). However, these putative structures lacked sufficient $\mathrm{G}+\mathrm{C}$ content, suggesting that transcription termination is ineffective in the typical rho-independent fashion (Farnham \& Platt, 1981).

\section{RT-PCR DNA assay}

M. hyopneumoniae chromosomal DNA template was used as a negative control to assess the relative quantity index and accuracy of the qRT-PCR. In this assay, no significant differences between the six target sequences in region ig072 would be expected since the relative concentration of template should be the same across all areas of the IG and flanking regions. As expected, no differences were observed across all pairwise comparisons of six probes (15 tests of significance) at $P<0.01$ (Fig. 1a).

\section{RT-PCR IG regions assay}

Thirty-five IG regions greater than 500 bases in length were identified in our analysis, and from these, five were selected for qRT-PCR analysis based on the direction of transcription of the flanking ORFs. Representative regions of the four possible read direction combinations were selected with the following criteria in mind: (1) one IG region had both flanking ORFs on the forward strand (ig-072); (2) one IG region had both flanking ORFs on the reverse strand (ig282); (3) one region had both flanking ORFs transcribed into the IG region (ig-682); and (4) two regions had both flanking ORFs divergently transcribed away from the IG region (ig-106, ig-684). A sixth region of less than $500 \mathrm{bp}$ comprising the upstream region of $d n a K$ was examined as a positive control; this region is known to undergo significant upregulation during heat shock (Madsen et al., 2006b).

The five IG regions were assayed using two biologically distinct RNA samples. The pattern of relative transcription was nearly identical for both RNA samples, as can be seen in Fig. 1(b-f). Statistically significant differential levels of transcription were detected in four out of five regions. The most common difference was a drop-off in RNA levels at ORF/intergenic transitions. In only one region, ig-682, did we fail to detect a signal by qRT-PCR at one of the six target sequences. To confirm the primers and reaction conditions for this target sequence, for other selected primer sets (Table 1), and for the entire ig-072 region, $M$. hyopneumoniae chromosomal DNA was used as a template in the qRT-PCR, which gave positive reactions for every primer set tested (data not shown). In Fig. 1(a) we show an example of the chromosomal DNA control reactions for region ig-072.

Analysis of region ig-072 (Fig. 1b) shows that for both biological samples, the qRT-PCRs that bridge the transition from ORF to IG region (primer sets 2 and 5) have significantly lower amounts of transcripts $(P<0.02)$ than the adjacent ORF regions (primer sets 1 and 6) although some signal was detected. There was no significant difference between the IG probes (primer sets 3 and 4) and the two ORF probes (primer sets 1 and 6). Following the decrease in transcript levels at the end of mhp072 (primer set 2), there was an increase in transcript levels across the IG region (primer sets 3 and 4) followed by another decrease at the ig-072-mhp073 junction (primer set 5$)$. This increase was statistically significant $(P<0.01)$ between primer sets 2 versus 3 and 4 for one of the two RNA samples and the decrease was significant for both RNA samples (primer sets 3 and 4 versus 5).

Region ig-106 (Fig. 1c), which contains sequences between divergently transcribed (outward) genes mhp106 and mhp107, shows a different pattern of transcription. The IG region (primer sets 2 and 3 ) demonstrates a statistically indistinguishable level of transcription $(P>0.1)$ relative to mhp107 (primer set 5) for one of the two RNA samples. Transcription in mhp106 (primer set 1), however, is significantly higher $(P<0.01)$ than that in the IG region (primer sets 2 and 3 ).

Region ig-282 (Fig. 1d), which has both ORFs transcribed in the same direction on the reverse strand relative to DNA replication, also demonstrated IG transcripts. At the end of mhp283 (primer set 4), transcription decreased significantly $(P<0.0001)$ from levels within mhp283 (primer set 5) and then increased within the IG region (primer sets 2 and 
$(a)_{\text {ig-072 }}$

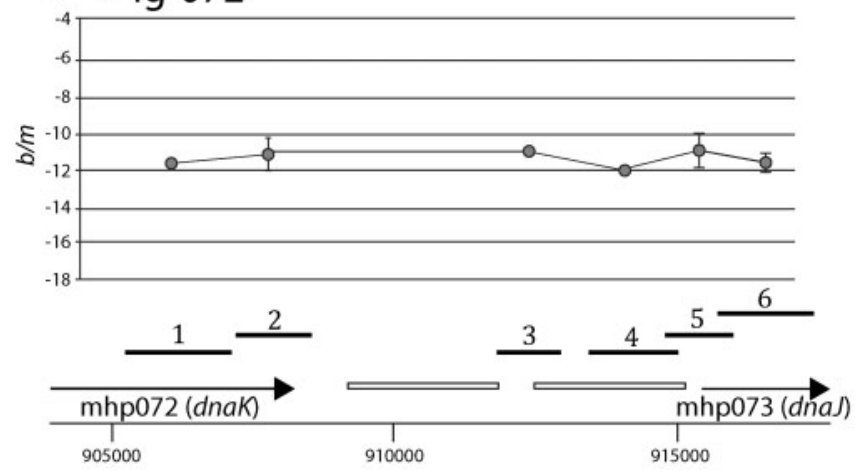

(C) ig-106
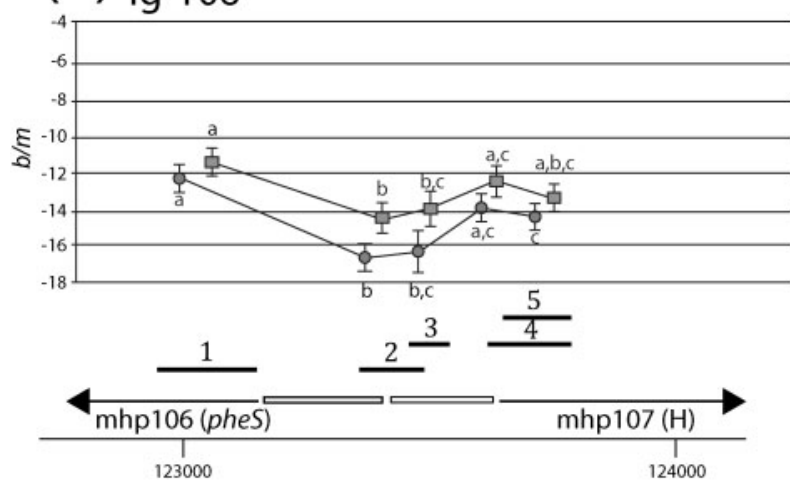

(e) $)_{\text {ig-682 }}$

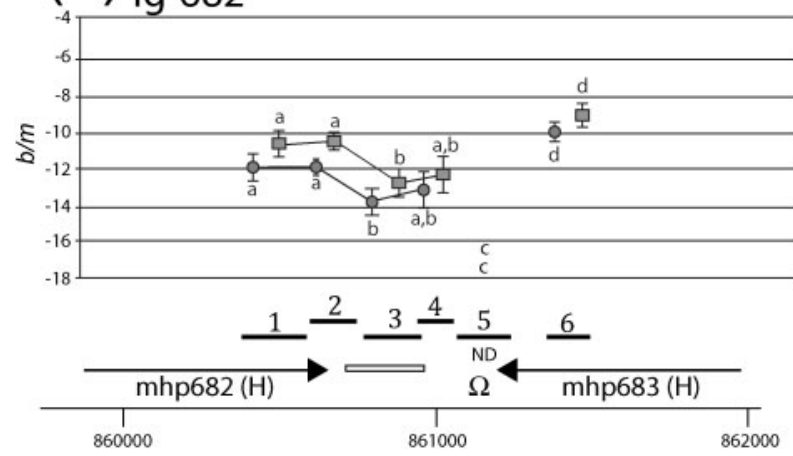

(b) ig-072

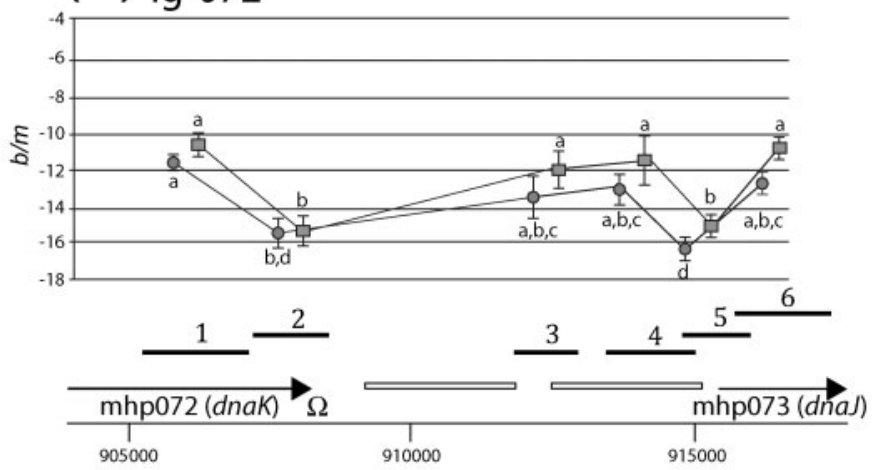

(d) ${ }_{\text {ig-282 }}$
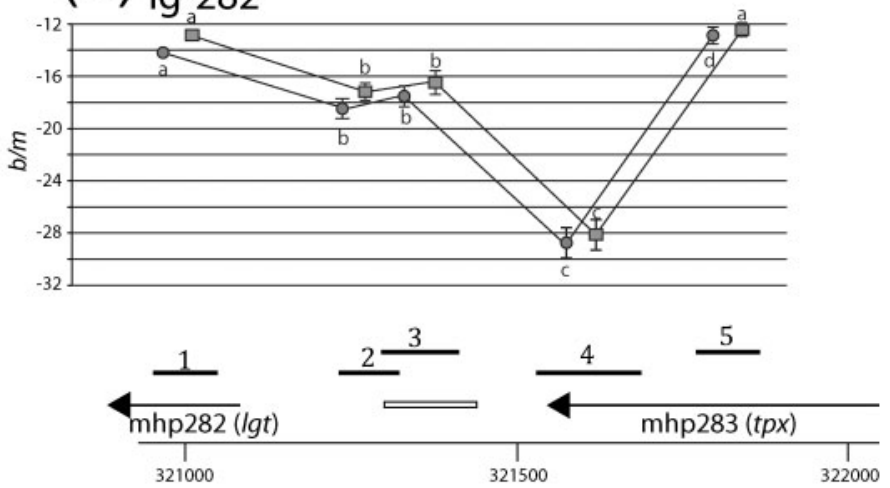

(f)

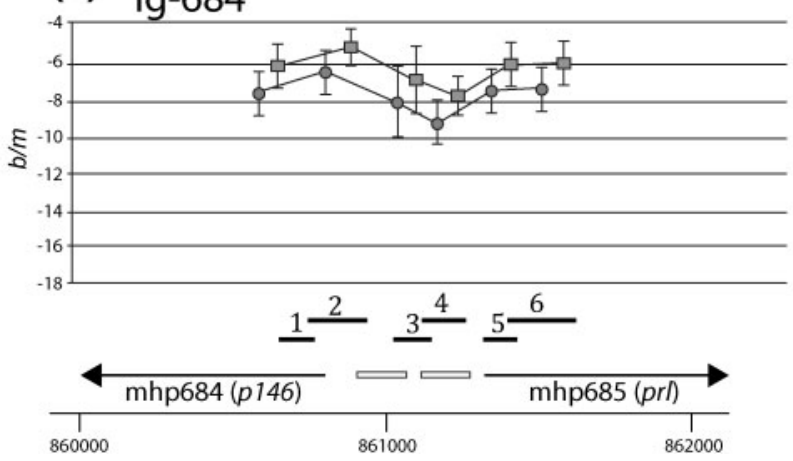

Fig. 1. $q R T-P C R$ analysis of $I G$ regions of $M$. hyopneumoniae. Shown are the relative quantity $(b / m)$ estimates of each qRT-PCR plotted vertically over the corresponding product location in the M. hyopneumoniae genome sequence given in base pairs. The data represent the relative quantity index estimate $\pm 2 \mathrm{SE}$ of the estimate. The IG designation is given at the top of each panel. The circles and squares depict results for the two biologically distinct samples. Each curve represents an independent RNA sample except for (a), where genomic DNA was used as a template for a positive control reaction. The letters indicate statistically significant differences. Points with the same letter are not significantly different $(P<0.01)$ for that independent RNA sample. The ORF designation and gene name are given for each flanking gene $(H$, hypothetical), and the arrows indicate the direction of transcription. The open bars indicate the location of PCR products used as probes in the microarrays. The filled bars indicate the qRT-PCR target sequences, and the numbers indicate the qRT-PCR primer sets described in Table 1 in that region. ND indicates that the probe was not detected in the assay. $\Omega$, putative stem-loop structure as predicted by SoftBerry FindTerm software.

3, $P<0.0001$ ). Transcription within mhp282 (primer set 1 ) was higher $(P<0.0001)$ than in the IG region (primer sets 2 and 3).
For region ig-682 (Fig. 1e), where flanking ORFs transcribe into the IG region, transcript levels between mhp682 (primer set 1) and the bridge region (primer set 2) were the 
same followed by a decrease $(P<0.01)$ in the IG region (primer set 3). No signal was detected with the ig-682/ mhp683 bridging region (primer set 5) even though the DNA template control was positive (data not shown). Transcription in mhp683 (primer set 6) was higher than that of all other areas within this region $(P<0.01)$.

The primer sets for region ig-684 (Fig. 1f) did not perform well, as indicated by the higher level of variation. This region is of the same type as ig-106, with both flanking ORFs being transcribed away from the IG region, but the region has a higher $\mathrm{A}+\mathrm{T}$ content $(83.3 \mathrm{~mol} \%)$, significantly contributing to difficulty in primer design. The trend in transcript levels of ig-684 is similar to ig-106, where the IG region had detectable but lower levels of transcription relative to either of the ORFs.

\section{RT-PCR analysis of the dnaK up- and downstream regions following heat shock}

The region surrounding $d n a K$ was studied as a positive control because both dnaK and dnaJ have been shown to be highly upregulated during heat shock (Chang et al., 2008; Madsen et al., 2006b). For this study, IG regions upstream (ig-070/071) and downstream (ig-072) of dnaK were assayed separately. The results can be seen in Fig. 2 .

The top graph in Fig. 2 shows the $\log _{2}$ fold change in transcription levels between control and heat-shock conditions. All target sequences in both regions indicate increased levels of mRNA under heat-shock conditions. This increase was statistically significant $(P<0.05)$ for all primer sets except for primer set 3 in ig-070/071 $(P=0.11)$. In region ig-070/071, the dnaK target sequence was the most upregulated: 13.38-fold higher amount of transcript than under normal growth conditions. All other target sequences in this region were upregulated about 2 -fold. In region ig-072, the mhp072 bridge target sequence had the highest level of upregulation (20.95-fold higher transcript levels), with $d n a K$ as the next highest level of transcript increase (7.26-fold). The rest of region ig-072 was upregulated 3-4-fold, except for the other bridge area with $d n a J$, which was upregulated about 5-fold.

The lower graph depicts the relative index $\left(C q^{\prime} / m\right)$ averaged across the three biological replicates within each treatment for both regions. Primer sets 1, 2 and 5 in region ig-070/071 have lower levels than primer sets 3, 4 and 6 $(P<0.01)$ for both treatment groups. The results for region
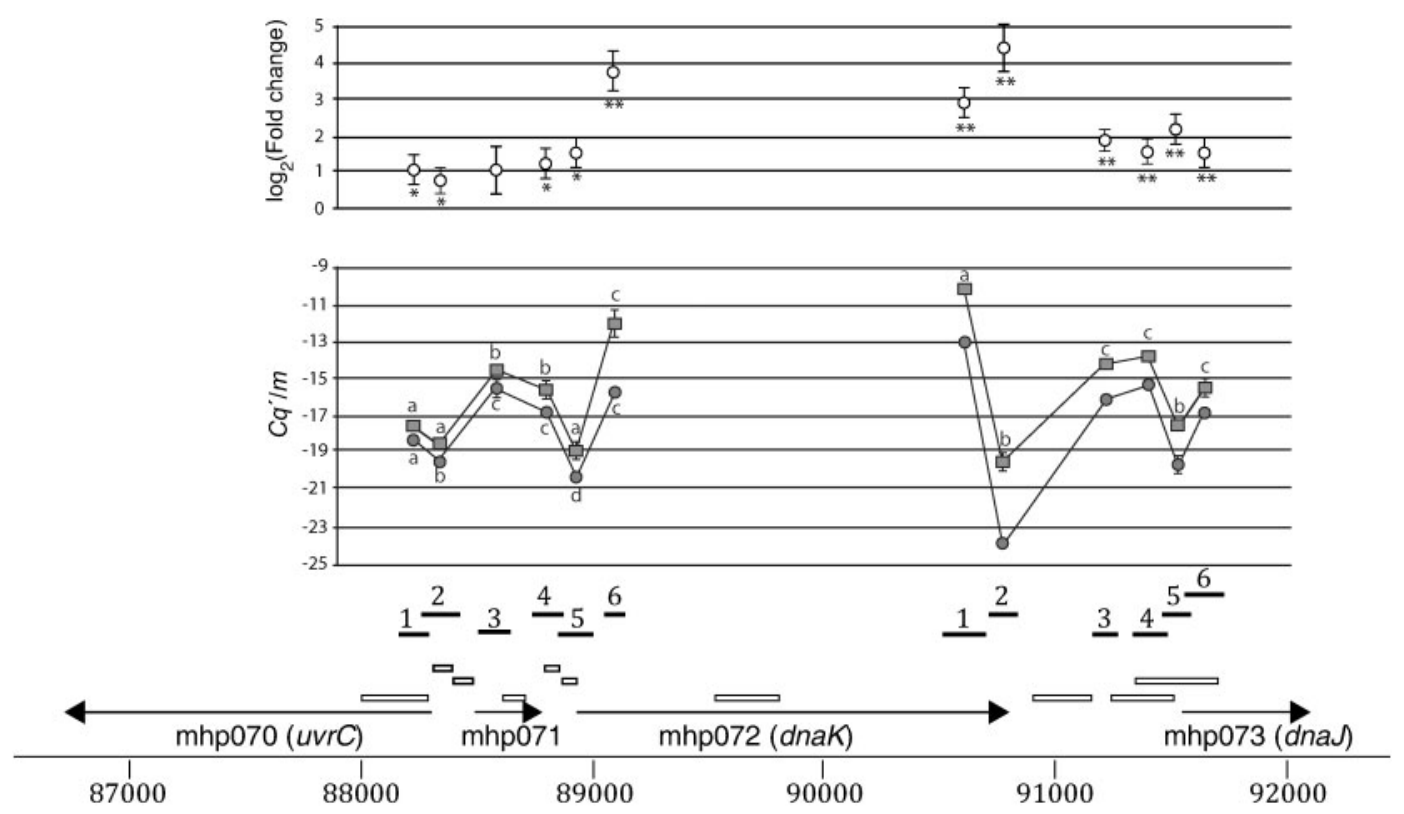

Fig. 2. Transcriptional analysis of $I G$ regions flanking $d n a K$ following heat shock. The map shows the corresponding region in the genome of $M$. hyopneumoniae given in base pairs. The ORF designation and gene name is given for each gene, and the arrows indicate the direction of transcription. mhp071 is a hypothetical gene. Open bars indicate the location of probes (PCR products or oligonucleotides) used in the microarrays. Filled bars indicate the qRT-PCR target sequences, and the numbers indicate the qRT-PCR primer sets described in Table 1 in that region. The upper panel gives the fold change (mean \pm 2 SEM) of triplicate samples. The asterisks indicate significant differences between the control and heat-shocked samples $\left(^{\star}, P<0.05 ;{ }^{\star *}\right.$, $P<0.01)$. The lower panel gives the relative quantity $\left(\mathrm{Cq}^{\prime} / \mathrm{m}\right)$ estimates of each qRT-PCR at the two temperatures. Control samples are represented by circles and heat-shocked samples by squares. Data represent the mean \pm 2 SEM of triplicate samples (note: most SEM bars are not visible due to being smaller than the plotted point). The letters indicate significant differences. Points with the same letter are not significantly different $(P<0.01)$ for that treatment. 
ig-072 are very similar to the results from the qRT-PCR region assay described above. For both treatment groups, the bridge areas have an appreciably lower level of transcription, and the IG region has levels of transcription closer to the dnaJ levels.

\section{DISCUSSION}

This study represents a global assessment of transcription within IG regions of the M. hyopneumoniae genome. We used both microarrays and qRT-PCR to assess transcription. The decision was made to limit our analysis to IG regions of $50 \mathrm{bp}$ or more using a combination of PCR products and oligonucleotide as probes. Of the IG regions in this set, 35 were large (>500 bp), requiring multiple PCR products or oligonucleotides for at least $50 \%$ coverage. Coverage was not $100 \%$ because of the inability to design unique primers or oligonucleotides in regions of high $\mathrm{A}+\mathrm{T}$ content.

We considered the possibility in our primer design that cross-hybridization between ORF transcripts and IG region probes could account for some of the signals we obtained, and without more extensive analysis could not totally discount that possibility. Our detailed analysis of five IG regions by qRT-PCR, however, showed no evidence of cross-hybridization effects. All melt curves from qRT-PCR assays indicated that a single product was generated per target sequence.

Our first study with the arrays was to determine the optimal conditions for generating cDNA for labelling reactions using two different primer sets. The hexamer subset was chosen based on their lack of binding to $M$. hyopneumoniae rRNAs and binding to annotated ORFs in the genome (Madsen et al., 2006b). It was initially thought that this primer set might not label RNAs from IG regions and consequently give false negative signals. The second primer set was a commercial random hexamer primer preparation that would label rRNAs representing about $90 \%$ of the total RNAs, diluting the signals on the arrays while also labelling IG region RNAs. Unexpectedly, both primer sets gave similar signals across both IG arrays (data not shown). Thus, we used both primer sets in our analysis of the IG regions transcripts. A posteriori, it seems that equal labelling efficiency with the two primer sets may be due to runoff transcription into IG regions.

Our results indicated that a large proportion of the IG regions, 321 out 343 (93.6\%), were transcribed. This was unexpected and indicated that M. hyopneumoniae does not strictly control transcription termination as previously suggested (Washio et al., 1998). These studies are supported by recent studies with $M$. pneumoniae in which IG transcripts on a global scale were also identified by tiling microarrays (Güell et al., 2009) and on a limited scale with Mycoplasma genitalium and M. pneumoniae (Benders et al., 2005). From a physiological standpoint, the lack of transcription termination seems energetically wasteful, suggesting that mycoplasmas may have important roles for these transcripts, such as the generation of small regulatory peptides or RNAs that have yet to be defined in these organisms. None of these IG regions had definable promoter-like sequences using current algorithms (data not shown), although promoters in mycoplasmas have not been fully defined by mutational analysis and could vary between species and not be recognized by current software. Stem-loop structures that may define rho-independent transcriptional termination sites are thought not to be important in mycoplasmas (Washio et al., 1998). Our analysis of the five IG regions and adjacent ORFs examined showed evidence of weak stem-loop structures in two regions, ig-072 and ig-682 (data not shown).

The five regions for qRT-PCR analysis were chosen based on size ( $>500 \mathrm{bp})$, direction of flanking ORF transcription (similar and divergent transcription) (Fig. 1) and in the case of $d n a K$ its known transcriptional control mechanism (Chang et al., 2008). The chosen IG regions also represented both DNA replication forks to rule out any replication effects on transcription. In most bacteria, the direction of transcription and DNA replication coincide in most genes. Even though this is not true for $M$. hyopneumoniae (Minion et al., 2004), we wanted to eliminate this possibility by choosing IG regions from both sides of the chromosome. Regions ig-072, ig-106 and ig-282 were associated with the right clockwise replication fork, and regions ig-682 and ig-684 with the left counterclockwise replication fork. Also, mhp107 and mhp684 are P97 paralogues (Adams et al., 2005; Minion et al., 2004), and mhp683 is a P102 paralogue. These proteins, along with other members of these families, are thought to play roles in adherence to swine epithelium, enhance colonization and thereby contribute to virulence (Adams et al., 2005; Burnett et al., 2006; Wilton et al., 2004, 2009), thus increasing our interest in them for defined analysis.

Before analysing the data, we considered two possibilities: an abrupt termination of transcription at the end of the flanking genes with the loss of bridging QRT-PCR products (mechanism 1) and a general decline in transcript levels indicating that RNA polymerase was slowly falling off the template in the IG region (mechanism 2). For regions with outward divergent transcription, we thought transcription would not occur. Our data indicate that both mechanisms occur along with a third mechanism: reinitiation of transcription within the IG region (mechanism 3).

Analysis of five IG regions showed a general trend of lower transcription in the IG region relative to the flanking ORFs (Fig. 1). It is important to note that none of these regions was potentially involved in an operon producing a polycistronic message. This implies that there is no obvious reason for continued transcription across the region. However, it is clear from these studies that transcription initiated (mechanism 3) within IG regions ig-072, ig-106 and ig-282 since downstream of the flanking ORF the level of transcripts increased significantly (Fig. 1b-d). This is 
independent of the direction of DNA replication; the genes flanking ig-072 and ig-282 are transcribed in the same direction but on opposite strands. There is a considerable reduction in transcript levels at the end of the first gene, a general rise in transcript levels in the IG region and increased transcription in the downstream gene. This implies a third mechanism, one in which transcription never truly ceases between genes and reinitiation of transcription occurs within the IG region before encountering the promoter of the second flanking ORF.

Region ig-682 differed, however. This is a region where flanking ORFs are inwardly divergently transcribed (Fig. 1e). From mhp682, transcription continued into the IG region, and it appeared that the RNA polymerase gradually fell off the template as it proceeded into the IG region (mechanism 2). This is the only region studied that showed the same transcript levels within both the gene (primer set 1) and the bridging region (primer set 2). For mhp683, however, there was an abrupt termination of transcription at the end of the gene (mechanism 1). This was the only gene in which transcription was completely terminated; most of the other genes showed decreased transcription levels in bridging regions but significantly higher levels of transcription within the IG region (Figs 1 and 2) or IG region transcripts continuing into the flanking ORF. Further analysis of the IG region adjacent to mhp683, however, failed to reveal a sequence suitable for rho-independent transcription termination as defined in other genetic systems; although a stem-loop structure could be identified just downstream of the left primer binding site for primer set 5 in ig-682 (Fig. 1e), the region was $\mathrm{A}+\mathrm{T}$-rich, lacking the characteristic $\mathrm{G}+\mathrm{C}$ content of a transcription terminator. If this structure serves the purpose of a termination signal in M. hyopneumoniae, it is unusual. Analysis of IG regions ig-072 at the end of mhp072 and ig-282 at the end of mhp283 revealed smaller stem-loop structures also lacking $\mathrm{G}+\mathrm{C}$ content, suggesting that transcription termination is ineffective in the typical rho-independent fashion.

In further analysis of the heat-shock gene $d n a K$ and its flanking regions by qRT-PCR, we observed a general increase in transcription across the entire region during heat shock (Fig. 2). This region was included because of previous studies demonstrating increased transcription of dnaK during heat shock (Chang et al., 2008; Madsen et al., $2006 \mathrm{~b}$ ), and positive control of transcription changes was thought to be informative. Interestingly, there was a significant difference between control and heat-shock samples with every primer set tested except one. Because transcript profiles between control and heat-shocked samples were identical in the IG regions ig-070/071 and ig-072, this suggests that increased temperatures have an overall positive effect on transcription, at least in this genomic region. As expected, there was a much larger increase in mhp072 (dnaK) transcripts (Chang et al., 2008; Madsen et al., 2006b), and previous studies showed that this gene is controlled by the heat-shock regulator HrcA
(Chang et al., 2008). Also as expected, there was a sharp decrease in transcription at the end of $d n a K$, but some transcript remained, suggesting that termination was not complete. We were also able to show that transcript levels vary between genes: mhp070 $(u v r C)$ had significantly $(P<0.01)$ lower transcript levels than mhp071 and mhp072 (dnaK) under both growth conditions.

In summary, these results indicate that IG regions within the M. hyopneumoniae genome are transcriptionally active. For many regions, transcription continues from flanking genes with a gradual reduction until the next promoter is encountered. For some regions, there is an internal initiation of transcription from ill-defined sequences. Most surprising were the observations that almost every IG region could be detected on the arrays, suggesting that IG regions in mycoplasmas may hold surprises with functions yet to be determined.

\section{ACKNOWLEDGEMENTS}

This study was funded partially by a grant from the Iowa Livestock Health Advisory Council from the College of Veterinary Medicine, Iowa State University, and from a US Department of Agriculture Formula Fund to F.C.M. We thank Jack Gallup and Michael Carruthers for technical assistance with the qRT-PCR assay, and Melissa Madsen for help with designing the probes, microarray assays and reviewing the manuscript. We also thank Stephen Windom and Sunny Gulati for their assistance in writing computer programs to process the data.

\section{REFERENCES}

Adams, C., Pitzer, J. E. \& Minion, F. C. (2005). In vivo expression analysis of the P97 and P102 paralog families of Mycoplasma hyopneumoniae. Infect Immun 73, 7784-7787.

Benders, G. A., Powell, B. C. \& Hutchison, C. A., III (2005). Transcriptional analysis of the conserved $f t s Z$ gene cluster in Mycoplasma genitalium and Mycoplasma pneumoniae. J Bacteriol 187, 4542-4551.

Burnett, T. A., Dinkla, K., Rohde, M., Chhatwal, G. S., Uphoff, C., Srivastava, M., Cordwell, S. J., Geary, S., Liao, X. \& other authors (2006). P159 is a proteolytically processed, surface adhesin of Mycoplasma hyopneumoniae: defined domains of P159 bind heparin and promote adherence to eukaryotic cells. Mol Microbiol 60, 669-686.

Casella, G. \& Berger, R. L. (2002). Statistical Inference, 2nd edn. Pacific Grove, CA: Duxbury.

Chang, L.-J., Chen, W.-H., Minion, F. C. \& Shiuan, D. (2008). Mycoplasmas regulate the expression of heat shock protein genes through CIRCE-HrcA interaction. Biochem Biophys Res Commun 367, 213-218.

Farnham, P. J. \& Platt, T. (1981). Rho-independent termination: dyad symmetry in DNA causes RNA polymerase to pause during transcription in vitro. Nucleic Acids Res 9, 563-577.

Fraser, C. M., Gocayne, J. D., White, O., Adams, M. D., Clayton, R. A., Fleischmann, R. D., Bult, C. J., Kerlavage, A. R., Sutton, G. \& other authors (1995). The minimal gene complement of Mycoplasma genitalium. Science 270, 397-403.

Friis, N. F. (1975). Some recommendations concerning primary isolation of Mycoplasma suipneumoniae and Mycoplasma flocculare, a survey. Nord Vet Med 27, 337-339. 
Gallup, J. M. \& Ackermann, M. R. (2006). Addressing fluorogenic realtime qPCR inhibition using the novel custom Excel file system 'Focusfield2-6GallupqPCRSet-upTool-001' to attain consistently high fidelity qPCR reactions. Biol Proced Online 8, 87-152.

Glass, J. I., Lefkowitz, E. J., Glass, J. S., Heiner, C. R., Chen, E. Y. \& Cassell, G. H. (2000). The complete sequence of the mucosal pathogen Ureaplasma urealyticum. Nature 407, 757-762.

Gottesman, S. (2005). Micros for microbes: non-coding regulatory RNAs in bacteria. Trends Genet 21, 399-404.

Güell, M., van Noort, V., Yus, E., Chen, W. H., Leigh-Bell, J., Michalodimitrakis, K., Yamada, T., Arumugam, M., Doerks, T. \& other authors (2009). Transcriptome complexity in a genomereduced bacterium. Science 326, 1268-1271.

Hutchison, C. A., III \& Montague, M. G. (2002). Mycoplasmas and the minimal genome concept. In Molecular Biology and Pathogenicity of Mycoplasmas, pp. 221-253. Edited by S. Razin \& R. Herrmann. New York: Kluwer Academic/Plenum Publishers.

Hyman, H. C., Gafny, R., Glaser, G. \& Razin, S. (1988). Promoter of the Mycoplasma pneumoniae rRNA operon. J Bacteriol 170, 32623268 .

Janis, C., Lartigue, C., Frey, J., Wrâoblewski, H., Thiaucourt, F., Blanchard, A. \& Sirand-Pugnet, P. (2005). Versatile use of oriC plasmids for functional genomics of Mycoplasma capricolum subsp. capricolum. Appl Environ Microbiol 71, 2888-2893.

Knudtson, K. L. \& Minion, F. C. (1993). Construction of Tn4001 lac derivatives to be used as promoter probe vectors in mycoplasmas. Gene 137, 217-222.

Knudtson, K. L. \& Minion, F. C. (1994). Use of lac gene fusions in the analysis of Acholeplasma upstream gene regulatory sequences. J Bacteriol 176, 2763-2766.

Lluch-Senar, M., Vallmitjana, M., Querol, E. \& Pinol, J. (2007). A new promoterless reporter vector reveals antisense transcription in Mycoplasma genitalium. Microbiology 153, 2743-2752.

Madsen, M. L., Nettleton, D., Thacker, E. L. \& Minion, F. C. (2006a). Transcriptional profiling of Mycoplasma hyopneumoniae during iron depletion using microarrays. Microbiology 152, 937-944.

Madsen, M. L., Nettleton, D., Thacker, E. L., Edwards, R. \& Minion, F. C. (2006b). Transcriptional profiling of Mycoplasma hyopneumoniae during heat shock using microarrays. Infect Immun 74, 160-166.

Madsen, M. L., Puttamreddy, S., Thacker, E. L., Carruthers, M. D. \& Minion, F. C. (2008). Transcriptome changes in Mycoplasma hyopneumoniae during infection. Infect Immun 76, 658-663.

Mare, C. J. \& Switzer, W. P. (1965). New species: Mycoplasma hyopneumoniae, a causitive agent of virus pig pneumonia. Vet Med Small Anim Clin 60, 841-846.

Minion, F. C., Lefkowitz, E. L., Madsen, M. L., Cleary, B. J., Swartzell, S. M. \& Mahairas, G. G. (2004). The genome sequence of Mycoplasma hyopneumoniae strain 232, the agent of swine mycoplasmosis. J Bacteriol 186, 7123-7133.

Musatovova, O., Dhandayuthapani, S. \& Baseman, J. B. (2006). Transcriptional heat shock response in the smallest known selfreplicating cell, Mycoplasma genitalium. J Bacteriol 188, 2845-2855.

Muto, A. \& Ushida, C. (2002). Transcription and translation. In Molecular Biology and Pathogenicity of Mycoplasmas, pp. 323-345. Edited by S. Razin \& R. Herrmann. New York: Plenum Press.

Neimark, H. C. (1986). Origins and evolution of wall-less prokaryotes. In The Bacterial L-Forms, pp. 21-42. Edited by S. Madoff. New York: Marcel Dekker.
Oneal, M. J., Schafer, E. R., Madsen, M. L. \& Minion, F. C. (2008). Global transcriptional analysis of Mycoplasma hyopneumoniae following exposure to norepinephrine. Microbiology 154, 2581-2588.

Pointon, A. M., Byrtr, D. \& Heap, P. (1985). Effect of enzootic pneumonia of pigs on growth performance. Aust Vet J 62, 13-18.

Pollack, J. D., Williams, M. V. \& McElhaney, R. N. (1997). The comparative metabolism of the mollicutes (Mycoplasmas): the utility for taxonomic classification and the relationship of putative gene annotation and phylogeny to enzymatic function in the smallest freeliving cells. Crit Rev Microbiol 23, 269-354.

Razin, S. \& Herrmann, R. (editors) (2002). Molecular Biology and Pathogenicity of Mycoplasmas. New York: Kluwer Academic/Plenum Publishers.

Razin, S., Yogev, D. \& Naot, Y. (1998). Molecular biology and pathogenicity of mycoplasmas. Microbiol Mol Biol Rev 62, 1094-1156.

Ross, R. F. (1992). Mycoplasmal disease. In Diseases of Swine, pp. 537-551. Edited by A. D. Leman, B. E. Straw, W. L. Mengeling, S. D’Allaire \& D. J. Taylor. Ames: Iowa State University Press.

Rozen, S. \& Skaletsky, H. J. (2000). Primer3 on the WWW for general users and for biologist programmers. Source code available at http://fokker.wi.mit.edu/primer3/. In Bioinformatics Methods and Protocols: Methods in Molecular Biology, pp. 365-386. Edited by S. Misener \& S. A. Krawetz. Totowa, NJ: Humana Press.

Schafer, E. R., Oneal, M. J., Madsen, M. L. \& Minion, F. C. (2007). Global transcriptional analysis of Mycoplasma hyopneumoniae following exposure to hydrogen peroxide. Microbiology 153, 3785-3790.

Szymanski, M., Erdmann, V. A. \& Barciszewski, J. (2003). Noncoding regulatory RNAs database. Nucleic Acids Res 31, 429-431.

Taschke, C. \& Herrmann, R. (1988). Analysis of transcription and processing signals in the $5^{\prime}$ regions of the two Mycoplasma capricolum rRNA operons. Mol Gen Genet 212, 522-530.

Taschke, C., Klinkert, M.-Q., Wolters, J. \& Herrmann, R. (1986). Organization of the ribosomal RNA genes in Mycoplasma hyopneumoniae: the 5S rRNA gene is separated from the $16 \mathrm{~S}$ and $23 \mathrm{~S}$ rRNA genes. Mol Gen Genet 205, 428-433.

Washio, T., Sasayama, J. \& Tomita, M. (1998). Analysis of complete genomes suggests that many prokaryotes do not rely on hairpin formation in transcription termination. Nucleic Acids Res 26, 54565463.

Waters, L. S. \& Storz, G. (2009). Regulatory RNAs in bacteria. Cell 136, 615-628.

Weiner, J., III, Herrmann, R. \& Browning, G. F. (2000). Transcription in Mycoplasma pneumoniae. Nucleic Acids Res 28, 4488-4496.

Weiner, J., III, Zimmerman, C.-U., Gohlmann, H. W. H. \& Herrmann, R. (2003). Transcription profiles of the bacterium Mycoplasma pneumoniae grown at different temperatures. Nucleic Acids Res 31, 6306-6320.

Wilton, J., Stewart, K. K., Minion, F. C., Young, P., Collins, A., Walker, M. F. \& Djordjevic, S. P. (2004). Domains in the carboxy-terminal of the cilium adhesin of Mycoplasma hyopneumoniae bind fibronectin and heparin. In 15th Congress of the International Organization for Mycoplasmology, p. 152. Athens, GA: International Organization for Mycoplasmology.

Wilton, J., Jenkins, C., Cordwell, S. J., Falconer, L., Minion, F. C., Oneal, D. C., Djordjevic, M. A., Connolly, A., Barchia, I. \& other authors (2009). Mhp493 (P216) is a proteolytically processed, cilium and heparin binding protein of Mycoplasma hyopneumoniae. Mol Microbiol 71, 566-582.

Edited by: C. Citti 